\title{
Interpolation of final geometry and result fields in process parameter space
}

\author{
Grzegorz Misiun ${ }^{1, a}$, Chao Wang ${ }^{1}$, Hubert Geijselaers ${ }^{1}$ and Ton van den Boogaard ${ }^{1}$ \\ ${ }^{1}$ University of Twente, Section of Applied Mechanics, Enschede, Netherlands
}

\begin{abstract}
Different routes to produce a product in a bulk forming process can be described by a limited set of process parameters. The parameters determine the final geometry as well as the distribution of state variables in the final shape. Ring rolling has been simulated using different parameter settings. The final state variable fields have been subjected to Principal Component Analysis. Interpolation of amplitudes in process parameter space gives a good representation of the fields and is useful as metamodel of the process.
\end{abstract}

\section{Introduction}

In engineering process design it is often needed to find an optimal solution for a given design parameters space. This type of research requires providing information about influence of different process parameters on final part results. Usually to avoid time and cost consuming experimental approach, numerical modelling is applied. Performing simulations significantly reduces costs and allows to give better insight into the process. Unfortunately in case of material nonlinearity which is characteristic for large variety of metal production processes numerical calculation may take even much more time than production itself. If there is a need for obtaining information from this process with large number of different parameter values it may be beneficial to construct its approximation response model known as surrogate model or metamodel.

Different mathematical techniques may be used for meta-modelling purposes. In [1] four different variants are compared for a simple FEM simulation: Response Surface Methodology, Proper Orthogonal Decomposition combined with Radial Basis Function, Moving Least Squares and Neighborhood Approximation. POD-RBF gives the best results when the number of sampling points is relatively small. This method will be used in this research as well.

In literature many examples regarding application of POD-RBF technique for metamodelling purposes may be found. Mostly they may be divided into two main categories. The most popular is inverse analysis where based on the system response compared to its metamodel some of the systems parameters such as material properties may be identified $[2,3,4]$. The second group consists of direct problems where based on POD-RBF methodology calculation time is improved by coupling it

\footnotetext{
$\overline{{ }^{a} \text { Corresponding author: g.s.misiun }} @$ utwente.nl
}

with FEM $[5,6]$ or where it is used to support the design process $[7,8]$.

In the present work the possibility of application of the POD-RBF technique is investigated to describe distributed FEM results for plastic metal forming process where large mesh distortions occur. Due to significant mesh deformations it is necessary to apply a mesh refinement algorithm during the simulations. This results in a newly created mesh with independent element distribution compared to its previous configuration. It means that simulations for different parameters will have different mesh number and configuration thus they cannot be directly used for metamodel creation. The possibility of describing part shape results independently from mesh distribution is investigated in this work for large deformation nonlinear problems.

\section{POD-RBF metamodeling methodology}

Three different methods are distinguished that can be used to perform POD: Karhunen-Loève Decomposition (KLD), Principal Component Analysis (PCA) and Singular Value Decomposition (SVD). The similarities and differences between them have been exhaustively described in [9]. In the present work decomposition is performed with use of the PCA methodology.

Apart from the possibility of applying different numerical approaches the effect of POD is always the same. It results in a subspace $\boldsymbol{\Phi}$ described by orthonormal basis vectors $\boldsymbol{\varphi}_{\mathbf{i}}$ with corresponding amplitudes $\mathbf{y}_{\mathbf{i}}$, for $k$ orthogonal vectors:

$$
\mathbf{X}=\sum_{i=1}^{k} \phi_{\mathrm{i}} \mathbf{y}_{\mathbf{i}}=\boldsymbol{\Phi} \mathbf{Y}
$$

The snapshot matrix $\mathbf{X}$ is created by collecting and concatenating data from simulations with different process parameters called snapshot vectors $\mathbf{x}_{\mathbf{i}}$. Before 
decomposition data should be preprocessed to have zero mean.

In case of PCA based decomposition a subspace $\boldsymbol{\Phi}$ is found by performing an eigendecomposition of a matrix created based on $\mathbf{X}$. Assuming that the snapshot matrix is a rectangular $m \times n$ matrix, two different matrices may be used for decomposition depending on the size of $m$ and $n$ :

- when $m>n$, the correlation matrix $\mathbf{D}=\mathbf{X}^{\mathbf{T}} \mathbf{X}$

- when $m<n$, the covariance matrix $\mathbf{C}=\mathbf{X X}^{\mathbf{T}}$

should be used. In both cases an eigendecomposition is performed. In this process it is stated that for any $p \times p$ square matrix A eigenvectors $p \times p \mathbf{V}$ can be found such that:

$$
\mathbf{A V}=\boldsymbol{\Lambda} \mathbf{V}
$$

$\boldsymbol{\Lambda}$ is a diagonal matrix storing $\lambda_{i}$ - the eigenvalues of matrix $\mathbf{V}$. Depending on $m$ and $n$ different matrices for $\mathbf{A}$ are used and their eigenvalues and eigenvector are obtained. Then for creation of a subspace $\boldsymbol{\Phi}$ simply eigenvectors $\mathbf{V}$ are used.

Next step of decomposition is to obtain the amplitudes $\mathbf{Y}$ corresponding to the new basis:

$$
\mathbf{Y}=\boldsymbol{\Phi}^{\mathrm{T}} \mathbf{X}
$$

After the decomposition, model reduction is performed. Based on eigenvalues $\lambda_{i}$ the first $l$ basis vectors and amplitudes are chosen to retain in a metamodel. The eigenvalues are sorted in descending order. Each eigenvalue corresponds to the energy accumulated within given eigenvector. Usually most of the energy is held in the few first vectors so that the rest can be considered as redundant. In order to reduce the amount of unnecessary data the cumulative energy factor is used. The cumulative energy $e$ of the $l$ first eigenvectors from a total of $n$, can be described as sum:

$$
e=\frac{\sum_{i=1}^{l} \lambda_{i}}{\sum_{i=1}^{n} \lambda_{i}}
$$

According to this factor some threshold of cumulative energy is set above which reduction is being performed, for example $90 \%$. The reduced set of vectors can be then used in (3), with assurance of the most optimal way of the data reduction.

Last step of metamodeling is interpolation of reduced amplitudes. Many different methods may be applied but the most common for POD metamodeling approach and used also in this research is Radial Basis Functions. With this method an approximation function is build based on the distance from known centers $-x_{i}$, and corresponding to them weights $-w_{i}$. The general approximation function for $N c$ centers takes form:

$$
f(x)=\sum_{i=1}^{N c} w_{i} \psi\left(\left\|x-x_{i}\right\|\right)
$$

The radial basis function $\psi$ can be any function that values depends on distance. In presented case the cubic function is being used:

$$
\psi\left(\mathrm{x}, x_{i}\right)=\left\|x-x_{i}\right\|^{3}
$$

The full method of obtaining weight values based on approximation function, data point locations and their values has been described in [10].

The metamodel created in that way may be used to predict values for new data points. Based on the interpolation functions, amplitudes values for a new data point may be obtained. When the amplitude values are known the whole process may be easily reversed with use of (1).

\section{Process description}

The process of preparing a preform for ring rolling can be divided into 4 stages - heat treatment, upsetting, forming and piercing (Fig. 1).

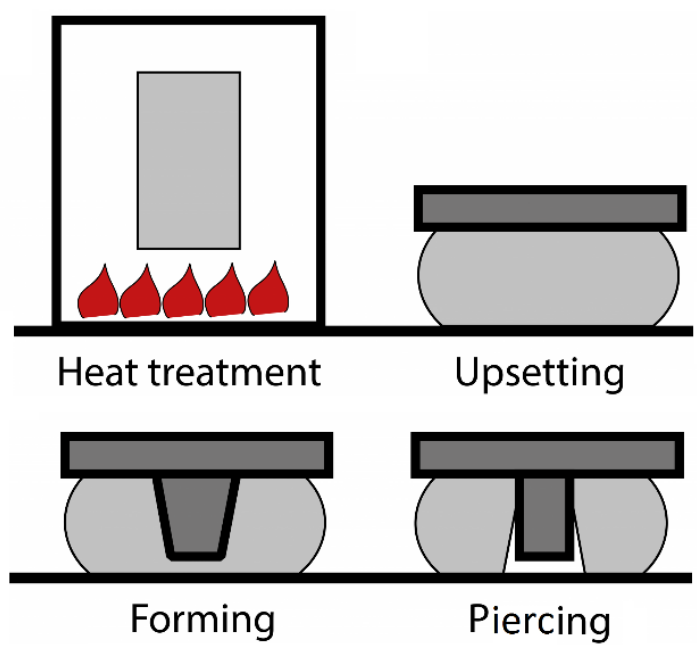

Figure 1. Stages of ring rolling operations: heat treatment, upsetting, forming and punching.

During these steps axisymmetrical, cylindrical initial billet shape changes. First, the part gets upset on the chosen height between two flat dies. Then the forming operation, takes place. The punch is pressed in the middle of a billet, displacing the material radially. The operation is carried out until the cavity bottom is $4 \mathrm{~mm}$ thick. Next the part is flipped over and piercing is performed. The thin $4 \mathrm{~mm}$ bottom is removed by the punch due to shearing and the hole in its middle is formed. Besides removing the bottom, piercing has one more important role. The flat tool surface to which punch is connected, is used for element flattening, because during forming and piercing the part shape may change. The punch moving distance is prescribed such, that its flat surface final height is $20 \%$ lower than the final upsetting height. It assures that element compression will take place. The part in its shape is then ready for the ring rolling operation $[11,12]$.

During the ring rolling process the initial preform is deformed simultaneously in radial and axial direction (Fig. 2). The main roll applies rotational movement on the outside diameter of the ring. The feed of the mandrel in main roll direction is responsible for radial deformation. The effect of radial deformation is reduced 
part thickness and increased diameter but also increased height. In order to control ring height two axial rolls, upper and lower are applied. Additional guide rolls assure process stability.

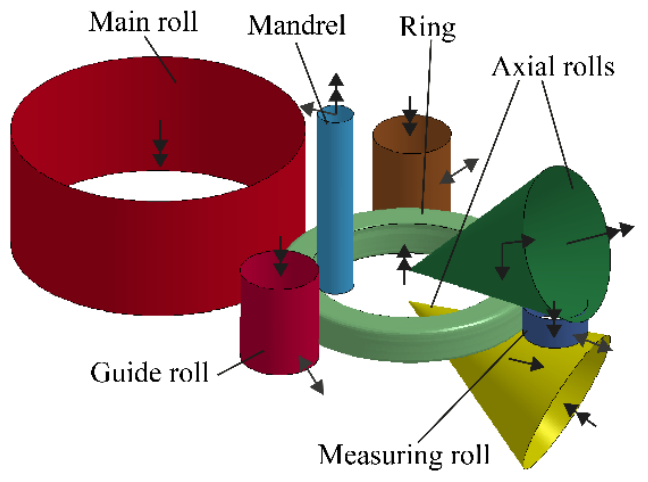

Figure 2. Model of ring rolling operation.

\section{Metamodeling of numerical analysis}

Numerical modeling is performed in LS-Dyna. The FEM simulations of preform forming are carried out with use of a 2D axisymmetrical model. The results of the final preform shape, after piercing are then mapped from $2 \mathrm{D}$ axisymmetrical state into $3 \mathrm{D}$ and then are used as an initial state for a ring rolling process simulation [11]. During the ring rolling process a large number of radial and axial deformations is applied until the final part geometry is reached.

In the presented work two different metamodels have been created. The first one provides information about shape and distributed part results for the preform stage. There are two parameter settings varied in this model: the part initial height and the height after upsetting. Due to constant volume constraint the part initial diameter varies with initial height. The height after upsetting defines its preform final height which is $80 \%$ of it.

The second metamodel gives information about the product after the rolling stage. Due to the fact that final part shape is always the same but only achieved with different tool paths the metamodel will focus only on the part distributed results. Beside initial part and upsetting height two additional parameters are implemented. The ring grow rate and the rolling path. The ring grow rate defines the rate of ring outer diameter growth during ring rolling process as a function of part shape [12]. The rolling path is a number that defines curve of a ring rolling deformation. It may vary from -1 to 1 . When the number is equal -1 the ring is first fully formed in axial direction then radially, 0 means proportional height and thickness reduction rates and 1 stands for first radial and then axial reduction.

\subsection{Preform metamodel}

As mentioned for the preform metamodel two variables are used: the initial billet height and the height after upsetting. In this parameter space 18 data points are defined for metamodeling purposes. Their spread is presented in Fig. 3.

Beside the data points used for creation of the metamodel, also the location of a test point which will be used for metamodel validation has been marked.

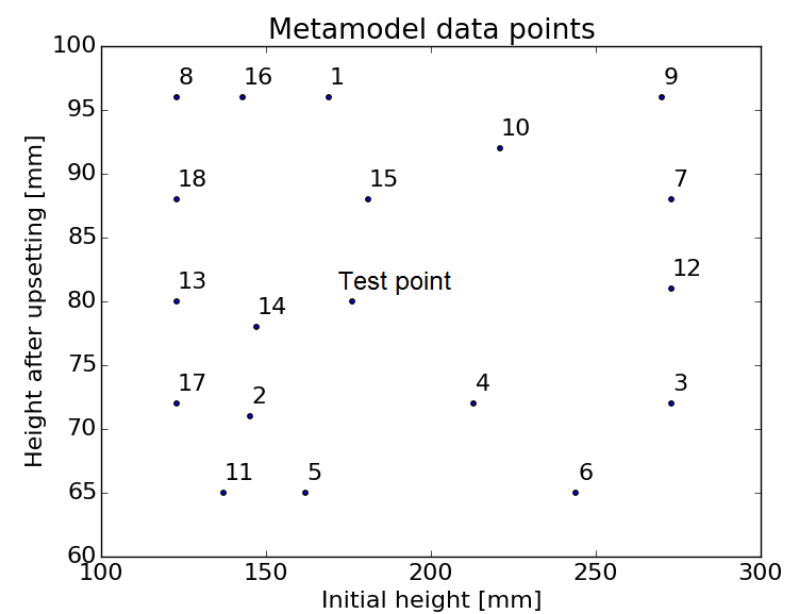

Figure 3. Data points used for creation of preform metamodel.

As mentioned in the presented metamodel there is a need of providing quantitative information about the part cross section shape and distributed results that is independent from mesh configuration. For this, a method is proposed which connects the part shape with its geometrical center coordinate, characteristic angles and lengths of lines from the center point to the part boundary (Fig. 4).

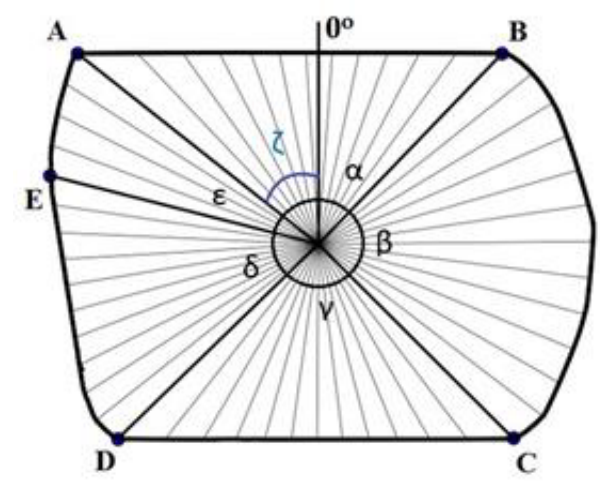

Figure 4. Visualization of method used for part description.

The part description begins with identification of the geometrical center. Next 5 characteristic points responsible for describing shape are identified. Four of them, A-D are corner nodes. E is a characteristic node located at the sharp corner, which is created during the piercing operation. The corner nodes assure that location of sampling points will change with the change of part dimensions. On the other hand a necessity of the point at the sharp corner $\mathrm{E}$ is dictated by inconsistency of shape correlation between different upsetting heights resulting from same shape of the forming stamp used for second, forming operation (length EA is always the same). The role of $\mathrm{E}$ is to prevent unnecessary data loss.

Based on this set of points the cross section is divided into 5 slices and the information about their size is stored as polar coordinates. Five angles describing size 
of every slice are stored. Additionally angle $\zeta$ is introduced. It describes angle between point $A$ and the vertical. It locates shape position in space. Last stage of part description consists of dividing each of slice by equally distributed lines from the center to part boundaries. The length of each line is stored which combined with other information is sufficient for part shape description.

Next data sampling point locations are determined. To assure sampling point independence from the element mesh grid and consistency between different shapes, the previously defined lines are used. On each line 10 points are equally distributed. At these points the distributed data results are sampled. Due to the fact that the distribution is based on polar coordinates, the density of points will increase with decreasing distance to cross section geometric center. That is why it has been decided to use every n-th data point within the data point row while the row distance decreases. According to this rule it has been decided for the farthest four rows to use 50 points, next four 25 points, then 10 points and finally 5 points (Fig. 5). The effect of reduction is 316 sampling points, while initial number of points was 501 . a)

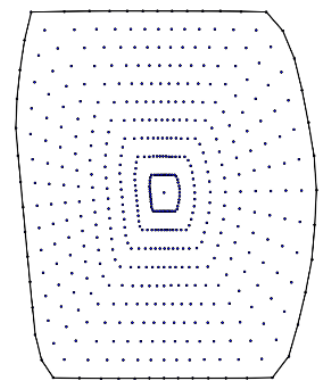

b)

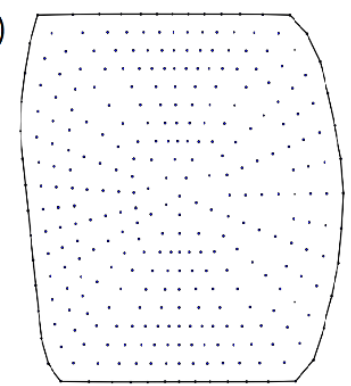

Figure 5. Distribution of sampling points a) before and b) after reduction.

Based on the presented method different distributed results may be sampled and used for metamodeling. It has been decided to create the metamodel in Python due to its large library of built-in functions. The procedure of metamodel construction is illustrated in Fig. 6.

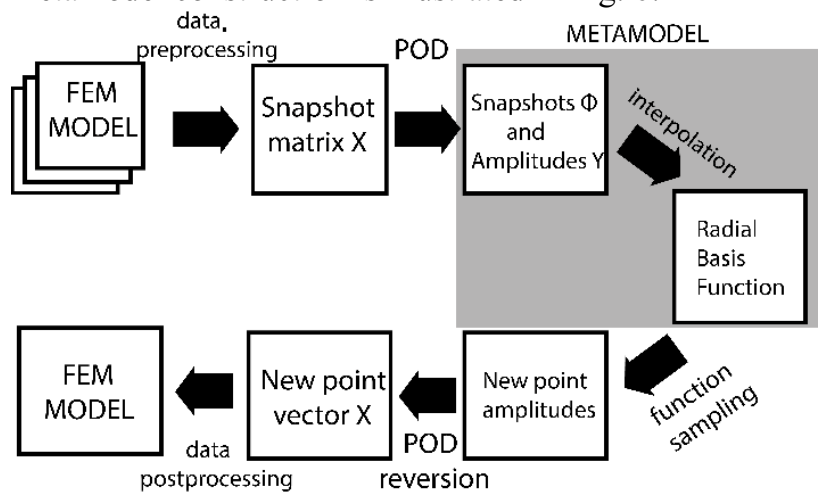

Figure 6. Process stages of POD-RBF preform metamodeling.

First the result from the FEM model are read into the program. The information is interpolated to allow its sampling according to sampling point locations. For this purposes quadric Radial Base Functions are used with centers corresponding to integration point locations. From the result fields obtained in this way, data is gathered and collected in separate vectors called snapshots. Separate snapshots are stored for different type of results. Next same snapshots from different simulation parameters are gathered and concatenated into one snapshot matrix $\mathbf{X}$. The data in this form is reduced by POD and then chosen amplitudes are interpolated with use of RBF. From interpolation function amplitude values for new data point may be obtained and the process is reversed according to (1). The results stored in the form of parameters describing the new shape and sampling point values are then interpolated with $\mathrm{RBF}$ and results obtained this way may be used for new FEM model creation.

In case of preform metamodeling investigation of 9 distributed results is performed: 4 stress components, plastic strain, 3 damage indicators and temperature. Separate POD decomposition has been performed for each of them and for the vector describing shape. Eigenvalues of each mode (Fig. 7) and cumulative eigenvalues energy (Fig. 8) for each of the decompositions is presented.

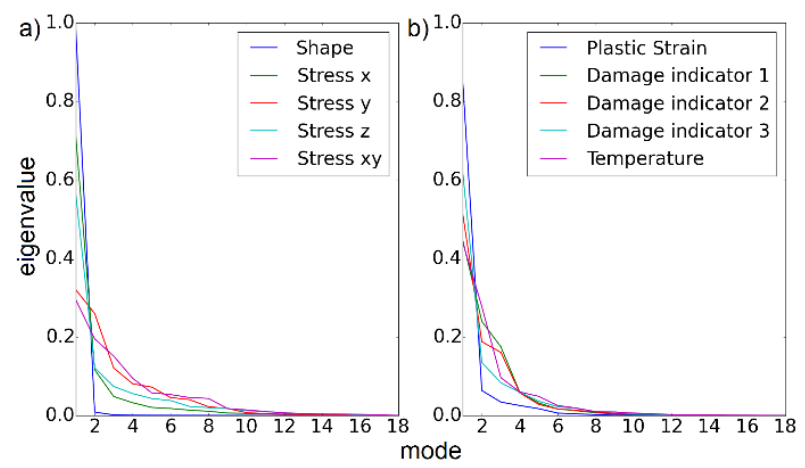

Figure 7. Eigenvalue of each mode for a) shape and stress results, b) plastic strain, damage indicators and temperature.

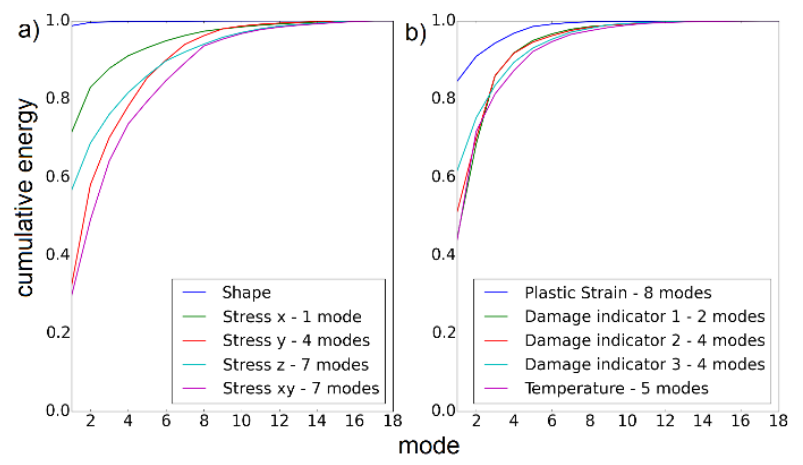

Figure 8. Cumulative eigenvalues energy of each mode for decomposition of a) shape and stress results b) plastic strain, damage indicators and temperature.

It can be noticed that in every case only first few modes contain most of the data information. According to cumulative energy a threshold $e$ from equation (4) equal to $90 \%$ of total decomposition energy has been set and applied for data reduction. Exception has been made for shape decomposition and plastic strain. In their cases first mode and first two modes over $90 \%$ of total energy. It has been established that this number vectors is to less to provide correct information about results and minimum value of required modes has been set to 3 . Number of 
first modes which contains $90 \%$ of total energy for different results type is listed in legend of figure 8 .

Creation of a metamodel requires amplitudes interpolation in reduced subspace. For this purpose the RBF are used. To check correctness of results, smoothness of interpolation functions has been checked for every type of results. As an example, the interpolation functions for the shape discretization are presented at figure 9 . a)

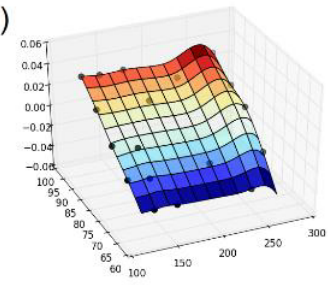

c)

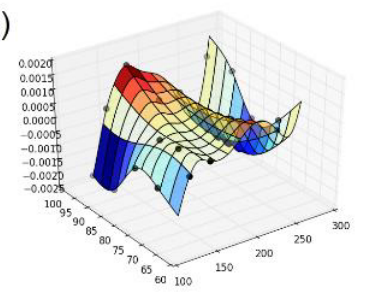

b)

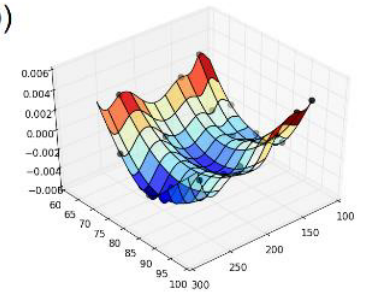

point locations from numerical model has been obtained and compared to metamodel. A global error measure is proposed:

$$
E_{g}=\sqrt[2]{\frac{\sum_{i=1}^{m}\left(x_{i}-\bar{x}_{i}\right)^{2}}{\sum_{i=1}^{m}\left(x_{i}-\mu_{x}\right)^{2}}}
$$

where:

$$
\begin{array}{r}
m \text {-number of sampling points } \\
x_{i}-\text { result values obtained from numerical model } \\
\bar{x}_{i} \text {-result values obtained from metamodel } \\
\mu_{x}-x_{i} \text { mean value }
\end{array}
$$

A summary of cumulative error different result types is presented in table 1:

Table 1. Relative cumulative error of preform metamodel

\begin{tabular}{|c|c|c|c|}
\hline Result type & $\mathrm{E}_{\mathrm{g}}[\%]$ & Result type & $\mathrm{E}_{\mathrm{g}}[\%]$ \\
\hline Stress $\mathrm{x}$ & 36.22 & Damage ind. 1 & 34.21 \\
\hline Stress y & 89.15 & Damage ind. 2 & 48.41 \\
\hline Stress $\mathrm{z}$ & 37.47 & Damage ind. 3 & 8.84 \\
\hline Stress xy & 109.94 & Temperature & 32.41 \\
\hline Plastic strain & 16.88 & \multicolumn{3}{|l}{} \\
\hline
\end{tabular}

Beside the cumulative error, a local relative error distribution is calculated:

$$
e=\frac{x_{i}-\bar{x}_{i}}{\sqrt{\frac{1}{m} \sum_{i=1}^{m}\left(x_{i}-\bar{x}_{i}\right)^{2}}}
$$

It can be noticed that for every type of results the smoothness of interpolation function decreases with increasing mode number. This seems correct and can be explained by the decreasing covariance of the data.

For results verification FEM calculation for the test data point (Fig. 2) has been performed. Results for the same parameters have been obtained from metamodel. Both result fields are compared. As the results before interpolation are preprocessed the error comes not only from data reduction but also from the method applied for sampling points location. In presented research the focus is only on the error coming from model reduction, assuming that different points numbers and distributions for better part description may be achieved. The part shape and results for stress in $\mathrm{x}$ direction are presented at figure 10 .
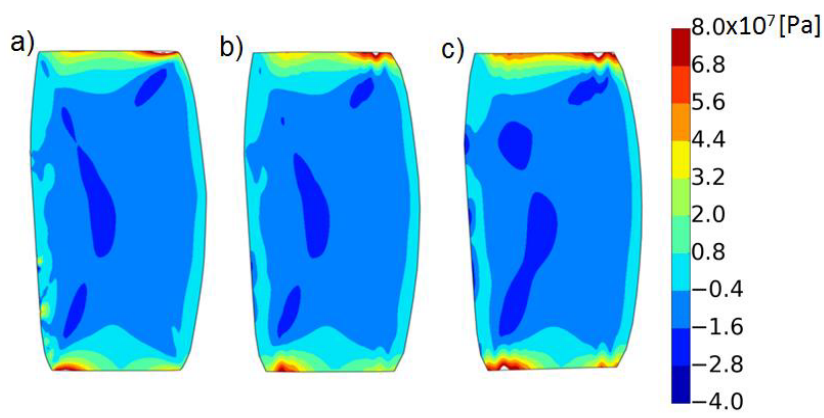

Figure 10. Comparison of stress in $\mathrm{x}$ direction for results obtained from a) numerical modeling, b) preprocessed data and c) metamodeling.

Visual inspection indicates high accuracy of metamodel results compared to standard FEM calculation. In order to verify results values at sampling
It has been decided that for an error distribution positive and negative values should be introduced for its better assessment. Error distribution for chosen results has been shown (Fig. 11). a)

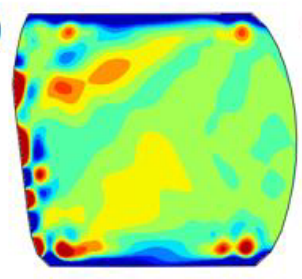

c)

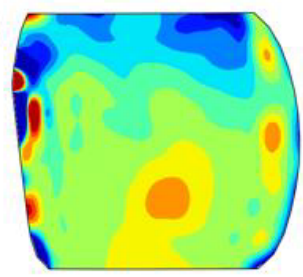

b)

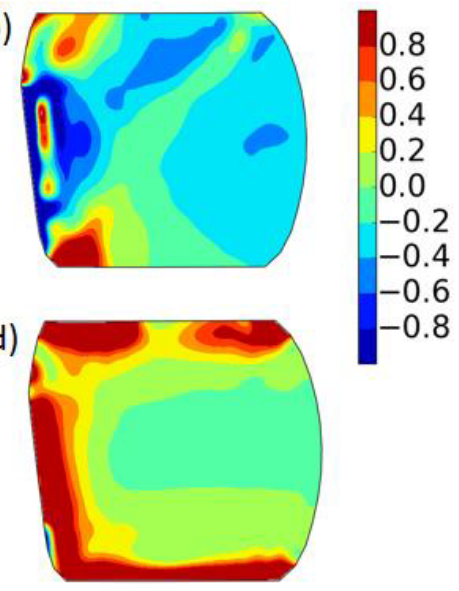

Figure 11. Error distribution for a) stress in $x$ direction, $b$ ) plastic strain, c) damage indicator 1 and d) temperature.

Beside metamodeling verification an algorithm has been developed which based on metamodel results creates input file for LS-Dyna. It uses commercial mesh algorithm and includes all of obtained results into file. Comparison of one of the results obtained for test point from numerical modeling and metamodel in LS-Dyna environment is presented in Fig. 12. 
a)

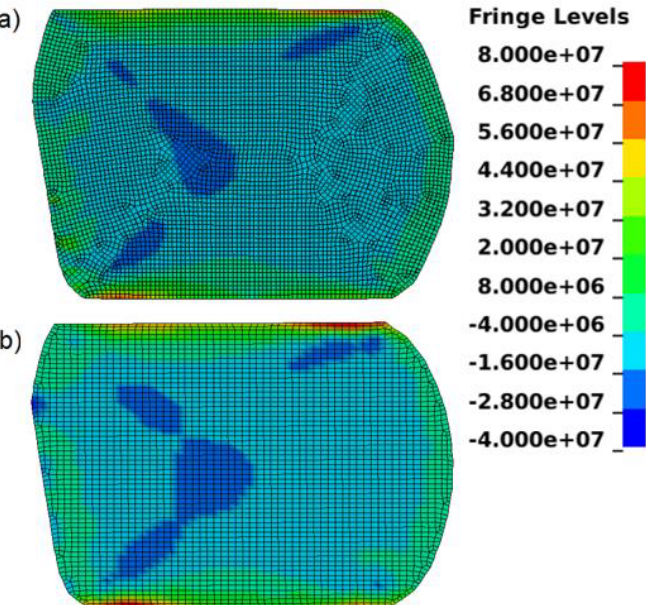

Figure 12. Comparison of results obtained for a) numerical modeling, b) metamodeling.

\subsection{Final results metamodel}

The metamodel for final results is created in 4 dimensional parameter space. In this case 17 data points have been used. Beside initial billet height and height after upsetting the ring rolling growth and rolling path are used. Due to thesmall number of data points their location has been carefully chosen to assure their optimal distribution.

The purpose of performing numerical calculations of ring rolling is to provide information about final distributed results according to different initial conditions and deformation histories. The final part shape is assumed to be constant. For creation of metamodel 5 of the most significant results has been identified - plastic strain, three damage indicators and the temperature field.

The results obtained from FEM ring rolling operation are in 3 dimensions. Despite spatial deformation history, overall part results are axisymmetrical. That's why it has been decided to perform metamodeling for a cross section instead of full three dimensional model.

Despite identical final tools positions for different types of parameters slight differences in the part shape may occur. It has been decided to compensate it by interpolation of the distributed results and describing the cross section shape by rectangle defined by maximum and minimum given cross section nodal points positions. Within this rectangle a uniform distribution of sampling points is generated (Fig. 13).

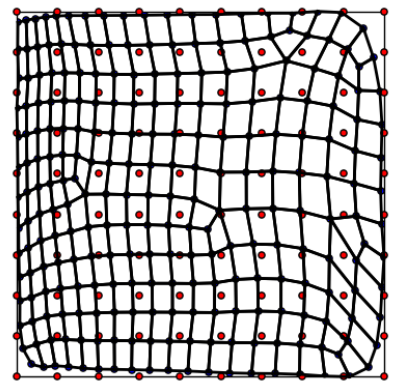

Figure 13. Exemplary cross section obtained from FEM model with sampling point distribution.
It can be noticed that some of the sampling points are located outside of the cross section geometry. It means that in their case sampled data will be obtained from extrapolated results and their values may under or overestimate real data. In case of metamodeling and from engineering points of view it does not matter. After ring rolling operation about $10 \%$ of the outside part cross section area is being removed by machining to assure good part quality. The points on the outside are used only to provide information about metamodeling and overall process quality but their values will not have influence on the final product.

Proper orthogonal decomposition of 17 data points for 5 distributed results has been performed. Based on their cumulative fluctuation energy (Fig. 14) number of reduced modes has been determined. Again the lower bound of 3 modes has been applied.
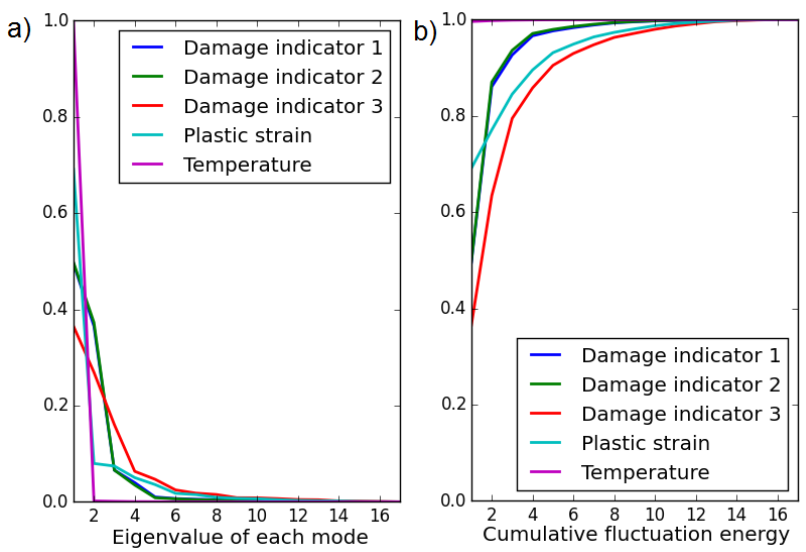

Figure 14. Second metamodel a) eigenvalues of each mode and b) cumulative eigenvalues energy.

For results verification numerical calculations for a new point has been performed. Point location has been carefully chosen to lay in a middle of the parameter range. Result of a numerical model has been compared with metamodel output for the same point. Field distribution for of the results has been plotted and compared.

Two of damage indicators - second and third and plastic strain has been chosen for more detailed check (Fig. 15). Both of them described damage as a contribution of hydrostatic tension within given plastic strain increment. The only difference between them is that the second assumes possibility of material healing.

For every decomposition global (7), and local (8) errors has been calculated. The summary of cumulative errors is presented in table 2 .

Table 2. Errors of final part metamodel

\begin{tabular}{|c|c|}
\hline Result type & $\mathrm{E}_{\mathrm{g}}[\%]$ \\
\hline Plastic strain & 34.88 \\
\hline Damage ind. 1 & 43.83 \\
\hline Damage ind. 2 & 43.21 \\
\hline Damage ind. 3 & 29.58 \\
\hline Temperature & 105.83 \\
\hline
\end{tabular}


a)

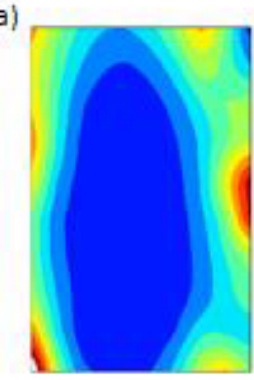

b)

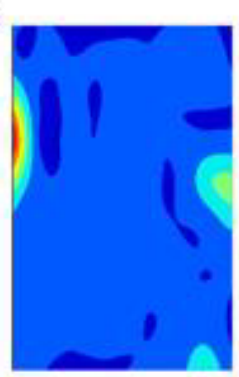

c)

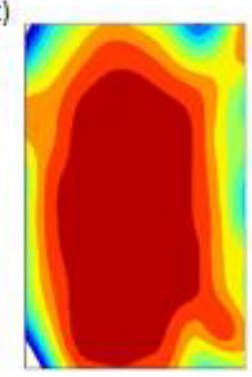

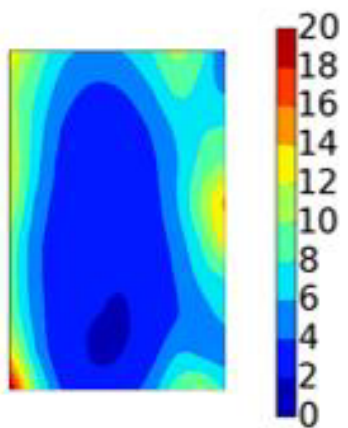
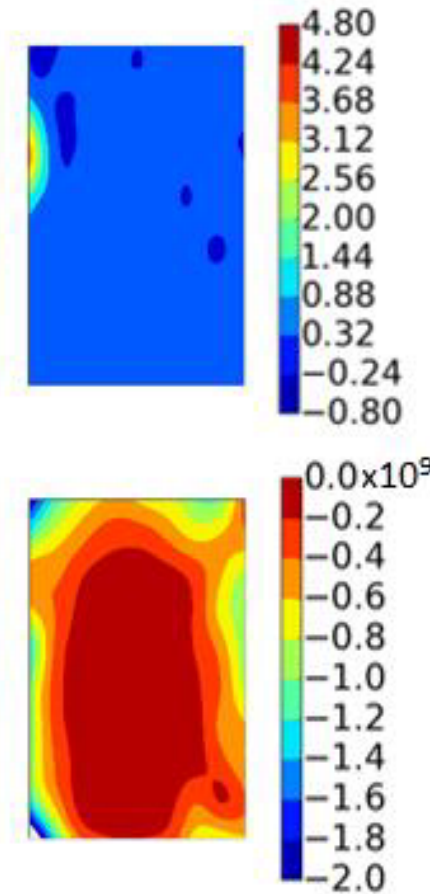

Figure 15. Result obtained from numerical model(left) and metamodel(right) for a) plastic strain, b) second damage indicator c) third damage indicator.

Moreover local error distribution for investigated results has been plotted and compared (Fig. 16).

\section{Results discussion}

Despite the fact that presented metamodels shows big similarity during their visual comparison their global error values are surprisingly big for both metamodels. It is probably caused by the sampling points inconsistency between different data points. Theirs localization does not fully correspond between different shapes and it results in preserving overall field trends but with slightly different local values.

Based on the result type different accuracy was achieved, what is indicated by different global error values. For preform metamodel the highest inaccuracy occurs when two stress fields are obtained. Stress in $y$ and xy direction. The probable reason for this is a large variance of these stress fields compared to other type of results. The stress results values usually show higher sensitivity for process parameters and they are affected by recent strain elastic deformation while cumulative results

a)

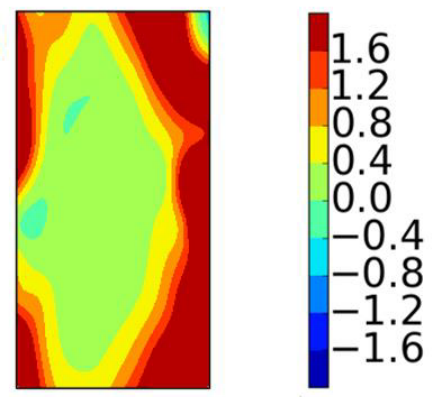

b)

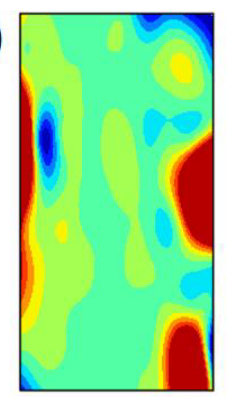

c)

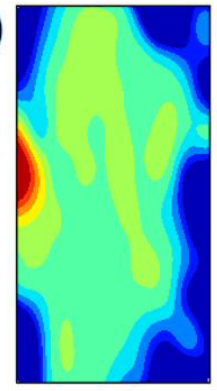

Figure 16. Local error distribution for a) plastic strain and b) second and c) third damage indicator

like plastic strain, damage indicators or temperature depend on whole deformation history. Moreover due to high field variance they may be more influenced by shape and sampling point location inconsistency. Visual comparison of fields distribution connected with local error distribution confirms it. Locally errors may be high but overall trends are preserved.

The results with smaller field variations like plastic strain, damage indicators and temperature shows higher accuracy. It may be noticeable that there is a difference in error level between first and second damage indicator compared to third. This is probably caused by their definition. The latter include material healing in them which mean that whole deformation history consist on it. The formers does not, which means that chosen deformation steps contributes to it which leads to the field with better outlined field. After inspection of local error distribution (Fig. 11c) it can be noted that biggest error occurs in upper part of element where shape inconsistency occurs which confirms this theory.

The enlargement trend of error values for the same results in the second metamodel may be noticed. It probably results from smaller number of data points compared to metamodel dimensions. Reduced number of data point per dimension may lead to poor interpolation function which will in consequence provide wrong amplitudes estimations. Visual comparison of different results again indicates big similarity of results despite the global errors. For plastic strain most of the field trends is preserved and overall results are good (Fig. 15a). Same tendency as for preform metamodel may be noticed in case of damage indicator. Material healing again leads to bigger nonlinearity of damage fields. Effect of it is that most of the result area oscillates around zero values which is harder to metamodel (Fig. 15b). The third 
damage indicator only contributes to damage which in consequence gives easier to describe trends (Fig. 15c).

By inspecting the local error distributions it may be noticed that in both metamodels higher error deviation occurs in case of local maxima and minima. The dominant behavior is described well but the error increases when the field departs from overall trend. It may be effect of metamodeling inaccuracy. Preservation of bigger number of modes connected with increasing number of data points should prevent that.

\section{Conclusion}

Presented results show that application of proper orthogonal decomposition connected with radial basis function may serve as an efficient tool for metamodeling of some distributed nonlinear FEM results. The overall field trends have been preserved in every case but global error values are high. Probable reason for that is inconsistency of sampling point locations between different data points. It is expected to increase model accuracy with different, deformation dependent point localization methodology.

Surrogate models for two stages of ring rolling forming - preform and final results, has been built and investigated. For highly nonlinear results the values of calculated errors are big. The reason for higher error values besides sampling points inconsistency lays probably within relatively small number of data points that do not describes nonlinear behavior fully. High local fields nonlinearity that with model subspace reduction leads to higher error values. It can be especially noted in second metamodel. Additionally first of investigated operations - forming of preform, shows shape inconsistency that influences some of results(damage indicators with healing).

Despite relatively high error values, visual inspection of results shows high correlation between data. Overall trends has been preserved and the fields extremum values lay within reasonable ranges. Created metamodels after some corrections, may be used as fast and computationally cheap method of result assessment.

\section{Acknowledgments}

This research was carried out under the project number M41.1.11418 in the framework of the Research Program of the Materials innovation institute M2i (www.m2i.nl). The industrial partner, SKF Group is gratefully acknowledged for the contributions to this research.

\section{References}

1. K. Khaledi, T. Schanz, S. Miro, J. Theor. Appl. Mech. 44, 45-54 (2014)

2. V. Buljak, G. Maier, Eng. Struct. 33, 492-501 (2011)

3. G. Bolzon, V. Buljak, Comput. Mech. 48, 675-687 (2011)
4. Z. Ostrowski, R.A. Bialecki, A.J. Kassab, Inverse. Probl. Sci. En. 16, 39-54 (2008)

5. A. Radermacher, S. Reese, Comput. Mech. 54, 677687 (2014)

6. R. A. Białecki, A.J. Kassab, A. Fic, Int. J. Numer. Meth. Eng. 62, 774-797 (2005)

7. M.J. Mifsud, S.T. Shaw, D.G. MacManus, Int. J. Numer. Meth. Fluids 63, 468-494 (2010)

8. E. Iuliano, D. Quagliarella, Comput. Fluids 84, $327-$ 350 (2013)

9. Y.C. Liang, H.P. Lee, S.P. Lim, W.Z. Lin, K.H. Lee C.G. Wu, J. Sound. Vib. 252, 527-544 (2002)

10. V. Buljak, Inverse Analyses with Model Reduction: Proper Orthogonal Decomposition in Structural Mechanics (Springer Verlag, Berlin, 2012)

11. C. Wang, H.J.M. Geijselaers, A.H. Van Den Boogaard, NUMIFORM 2013, 1532, 1014-1019 (2013)

12. C. Wang, H.J.M. Geijselaers, E. Omerspahic, V. Recina, A.H. Van Den Boogard, J. Mater. Process. Tech. 227, 268-280 (2016) 